\title{
Haemangioma: Surgery or Embolization?
}

Nor GM, Jaafar N. Haemangioma: Surgery or Embolization - a Case Report.

Annals Dent. Univ. Malaya 1995;2:33-36.

\begin{abstract}
The management of vascular lesions such as haemangiomas frequently present difficult choices between various treatment modalities. Circumstances may limit the number of options available for the surgeon. This article describes the circumstances, investigations, difficult choices, and eventual treatment of a case of a mandibular haemangioma. It is concluded that for a medium sized haemangioma such as in this case, in the absence of facilities to embolize it, surgical excision may be safely carried out provided that proper investigations and precautions have been conducted. An angiogram is of utmost important in determining the feeding vessels and is very important before any surgical procedure is attempted.
\end{abstract}

Key Words: Haemangioma, surgery, embolization.

"Ghazali Mat Nor
*Nasruddin Jaafar
* Department of Oral and Macillofacial Surgery
Faculty of Dentistry
University of Malaya
59100 Kuala Lumpur
Malaysia
** Department of Community Dentistry
Faculty of Dentistry
University of Malaya
59100 Kuala Lumpur
Malaysia

\section{INTRODUCTION}

Vascular lesions in the head and neck region is uncommon and very often difficult to manage. It may range from small haemangiomas to very large arteriovenous malformations. The haemangioma is a hamartomatous malformation which consists of numerous proliferating blood vessels. The condition may result in moderate to severe bleeding following a dental procedure such as biopsy or extraction.

Clinically it usually appears as a slow growing localized gingival growth which may bleed easily when minimally traumatized. It may blanch and be emptied on pressure. Pulsations may also be detected. The patient may experience slight tenderness, mild pain or discomfort from the lesion. The swelling is soft to firm in nature and not fluctuant. It does not cause any paraesthesia. The affected tooth or teeth may be displaced, mobile and manual pressure may cause the tooth to be pushed into the socket. If a tooth germ is affected, it would be displaced. The lesion if large enough may result in asymmetry of the jaw and face. Such a case requires extreme caution and a thorough investigation.

Central haemangiomas are more commonly found in females aged between $10-20$ years, $65 \%$ in mandible and more commonly in the posterior part. It is a great imitator because it may produce varied radiological features. It may appear as unilocular, multilocular or have a cyst-like appearance, some with hyperostotic border. The margin could be well or ill-defined. Occasionally root resorption can occur.

The possible modes of treatment are local surgery, ligation of the feeder vessel, cryosurgery, sclerosing agent, embolization, and radiation. A case of a large vascular lesion of the right mandible is reported and the management is discussed.

\section{CASE REPORT}

A 14 year old Malay female student was referred to the Oral and Maxillofacial Surgery Clinic for management of a haemorrhagic mass in October 1993. She first noticed a lump at the posterior part of lower right area of the mandible in August 1993. The lump was about $3 \mathrm{~cm}$ by $2 \mathrm{~cm}$ in size and situated buccal to the mandibular right first and second molars. The surrounding mucosa, free and attached gingiva looked nommal. The adjacent second molar was slightly mobile. The mass was expanding and there was little pain. Occasionally it bled when brushing and chewing.

During extraction of the lower right first molar which was carried out by the referring doctor and subsequent excision, moderate bleeding was encountered which was eventually arrested. The initial biopsy suggested that it was a pyogenic granuloma possibly because of numerous inflammatory cells and blood vessels.. A month later, the patient went to the same clinic again because the lump had reappeared and it was getting bigger. The surgeon then performed an aspiration biopsy which yielded blood. A provisional diagnosis of haemangioma was made and the patient was then referred to us for further management.

On general examination the patient was thin and tall. There was a slight swelling on the right side of the face. The patient was able to open the mouth but unable to properly close/occlude due to the mass interfering with occlusion.

Intra-orally, there was a large pedunculated lump 


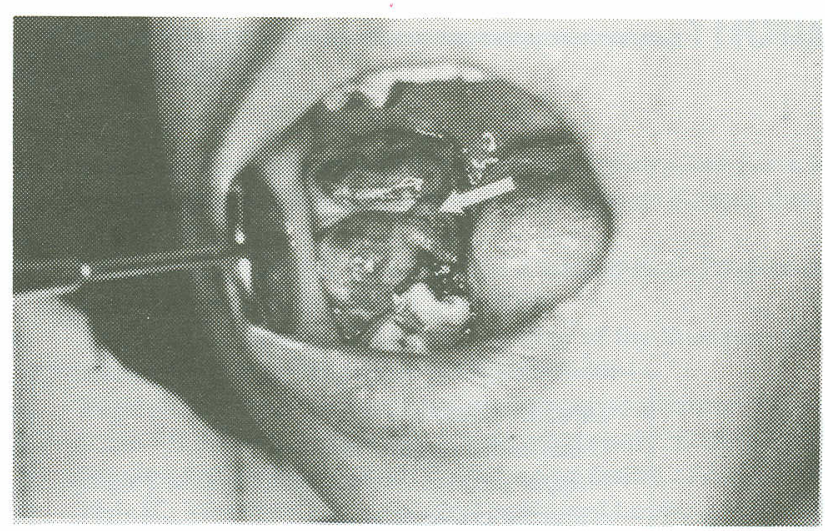

Figure 1. Clinical presentation showing the lesion to be growing from alveolar bone with surface ulceration and bleeding.

on the lower right molar region. The lump was oval with rough and granular surface. There were a few ulcerations on the surface with a few bleeding spots (Fig. 1). The buccal and lingual plates were expanded but not perforated. The patient was unable to chew food as the lesion was quite tender and occasionally caused bleeding. On palpation, the lump was firm and slightly tender but no bruit was detected. There was also a marked increase in salivation and halithosis. The cervical Iymph nodes were enlarged and tender. An aspiration was carried out which yielded blood. However incisional biopsy was strongly opposed as it might lead to troublesome bleeding.

Plain radiographic examination revealed multilocular radiolucent areas and the lower right third molar was displaced distally toward the ramus. The buccal and lingual bone plates of the mandible were expanded but the margin of the lesion was quite well defined (Fig. 2 and 3).

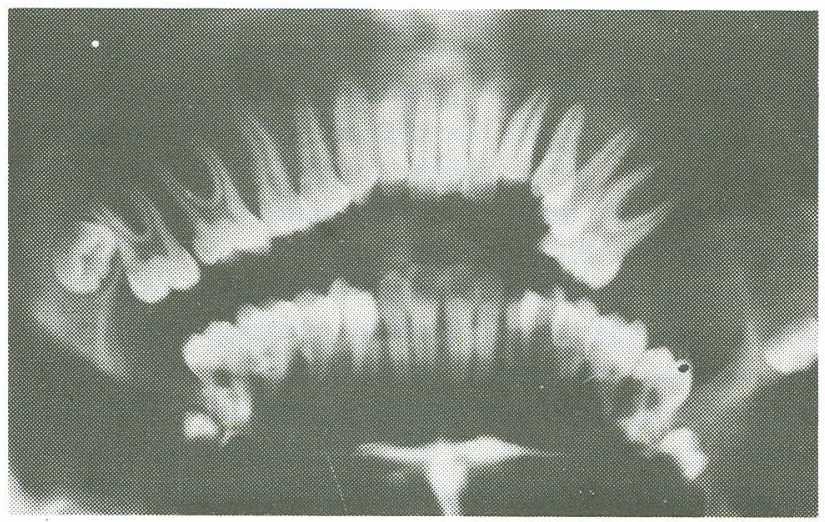

Figure 2. OPG radiograph showing extent of lesion and the displacement of the lowver right first and third molars.

Lateral facial angiogram was carried out using direct femoral catheterization (Seldinger Technique) and using an image intensifier. Angiography was done to determine the size, number and location of feeder vessels and to detect any other vascular spaces and its margin. The angiogram showed the vessel to be a normal looking inierior dental artery Fig. 4). It therefore helped to facilitate the choice of the trcatment.

Based on the radiographic and angiographic finding,

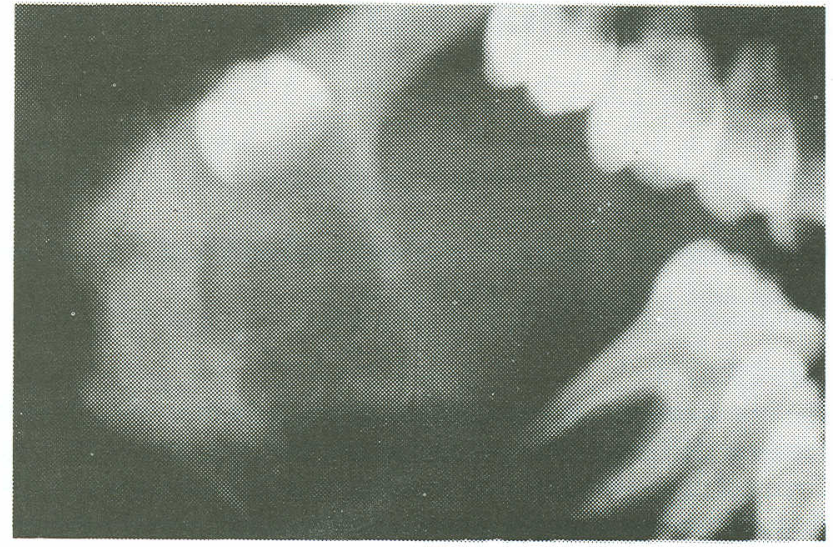

Figure 3. Lateral oblique radiograph showing multi-locular radiolucent areas between the lower first and third molar.

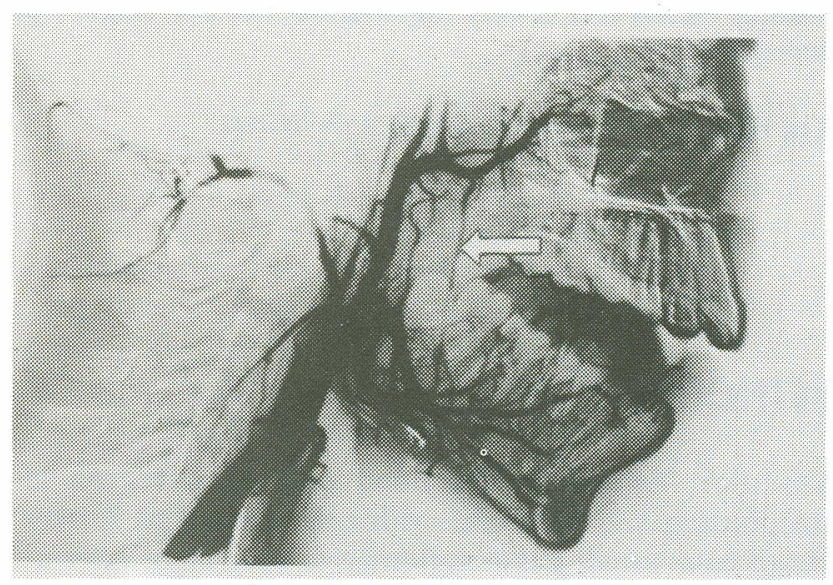

Figure 4. Substraction angiography marking the lesion and the surrounding vessels. (arrow shows inferior dental artery)

a decision was made to surgically excise the lesion. Meanwhile the lesion was getting bigger making eating and chewing very difficult. She was readmitted and routine haematological investigation showed that her haemoglobin level was low ( $9 \mathrm{gm} / 100 \mathrm{ml})$. Packed cells was transfused to raise the haemoglobin level prior to surgery.

Treatment consist of extraction of the lower right first molar and surgical excision of the lesion. There was moderate bleeding which was eventually arrested. The inferior alveolar nerve was identified and protected. The inferior alveolar artery was identified at the mandibular foramen and ligated to reduce bleeding. The defect was packed with gauze soaked with Whiteheads varnish. The lesion was reviewed and the pack regularly changed. Healing was good and uneventful (Fig. 5). Follow-up radiograph showed progressive reossification of the lesion.

The histological report showed that there were numerous vascular channels of various sizes, some with thickened wall filled with blood, somethrombosed and the tissue infiltrated with plasma cells, Iymphocytes and neutrophils with ulcerated mucosa and therefore diagnosed as inflamed haemangioma (Fig. 6). The patient was kept under surveillance in case other vascular lesions might' develop at other sites as reported by Akker(1). 


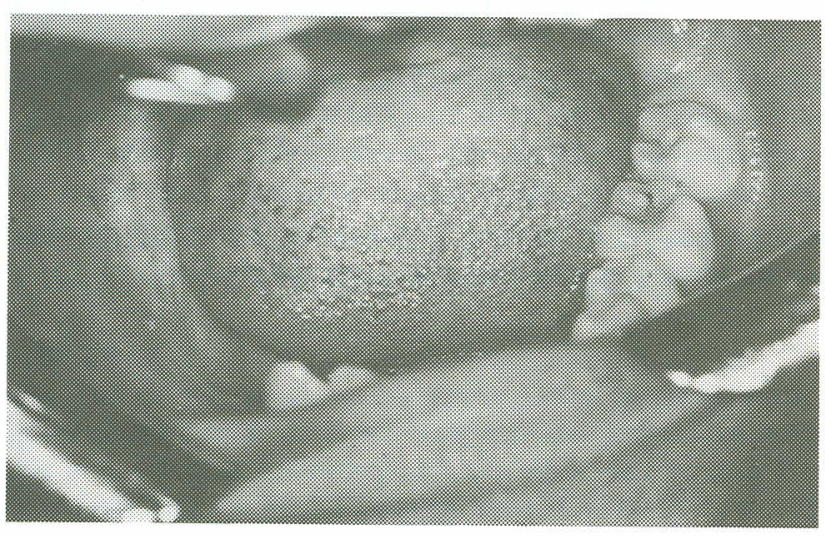

Figure 5. Complete healing following surgical excision.

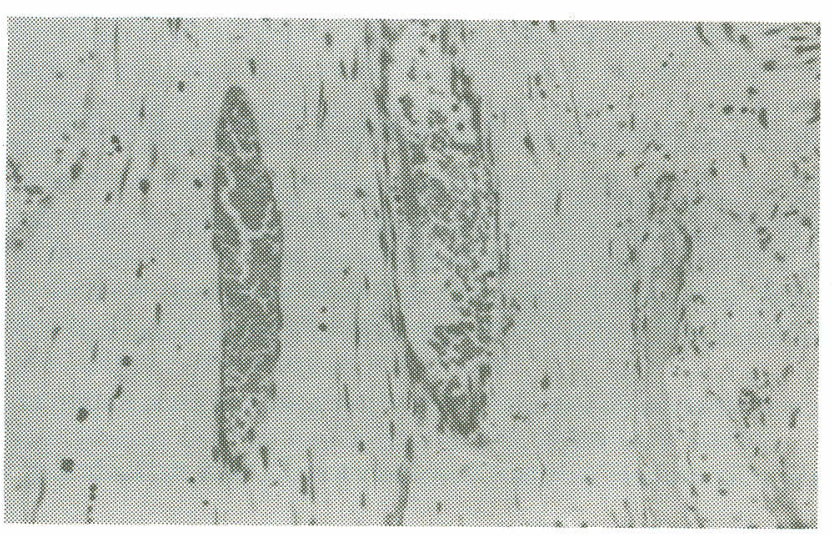

Figure 6. Histological appearance of lesion showing numerous vascular spaces and tissue infiltrated with plasma cells, lymphocytes and neutrophils.

\section{DISCUSSION}

This type of lesion is usually asymptomatic and may remain problem-free for years. It usually becomes clinically manifest in the second decade of life. In the asymptomatic case, a potentially dangerous situation exist since extraction and attempt at biopsy will result in severe bleeding. Ideally in order to get a definitive diagnosis an incisional biopsy should be carried out but in this case it was ruled out for fear of troublesome haemorrhage. This complication was reported in the previous extraction and excision by the referring doctor. A recognized but quite invasive method of investigation is the arteriogram, which was employed in the case. Other methods which are not so invasive which may be used to diagnose vascular lesions are the use of ultrasound and magnetic resonance imaging (MRI)(2).

Arteriovenous malformations in the mandible do not exhibit characteristic radiological features, being indistinguishable from other cystic or destructive process(3). The margin of these radiolucent areas vary from well-defined and corticated to ill-defined margin, the area of osteolysis gradually blending with the surrounding normal bony architecture. In a clinical situation where there is a red hemorrhagic mass with mobile teeth, it should warrant a high index of suspicion for the existence of an intraosseous vascular malformation(4). Such cases will require appropriate investigations before any surgical procedures such as biopsy or extraction is attempted.
Once the diagnosis of a vascular lesion is confirmed (by aspiration of blood, radiograph and angiography), a choice had to be made - either to do excision or to embolize it. The decision to do excision and curettage was opposed by another consultant who felt that the lesion should be embolized by a qualified and experienced radiologist. Unfortunately, due to unforeseen circumstances the latter was not possible. The fast growth rate of the lesion (within two weeks) made speech, chewing and swallowing very difficult. Although surgery has its potential complications, we proceeded with it with satisfactory results.

The decision to surgically excise the lesion was prompted by the following factors: i) The lesion was contained because it was growing (fungating in shape) from the alveolus and the lateral and lingual plates were intact as seen in the plain radiograph and extemal carotid angiogram, ii)The angiogram showed that there was no obvious large feeding vessel thus making surgery favourable. The lesion was fed by a normal looking inferior dental artery, a branch of the maxillary artery, iii) There were repeated episodes of bleeding especially at night and the patient often woke up with a blood clot in the mouth in the morning, and iv) The lesion continued to enlarge quickly making normal oral function difficult thereby warranting early surgery.

Superselective therapeutic embolization is also one of the treatment of choice for head and neck vascular lesions. This has been successfully done using unresorbable materials such as steel coil and Polyvinyl Alcohol (PVA)(7). Other agents that may be used for embolisation are Gelfoam, silicone spheres, methyl methacrylate, steel coils and autogenous muscle (8). Cyanoacrylate which sets on exposure to blood or serum could also be used. However, in view of the potentially serious complications which could take place, it is suggested that therapeutic external carotid artery embolisation should be restricted only to centers with experienced clinicians.

On the other hand, embolization has its advantages and complications. The advantages are to decrease the size of the lesion before surgery and to reduce haemorrhage during and after surgery. It may also be effective in producing total resolution(1). Initial embolisation can make the surgery less hazardous and it is best carried out by a fully trained and experienced radiologist as it carries with it a number of possible complications. This may include, at the site of the puncture, spasm of the vessel, haemorrhage, haematoma formation, pain and infection. Within the artery itself, there may be intimal damage or dissecting aneurysm. Other more significant complications are the inaccuracy in placement or reflux into the internal carotid artery resulting in cerebral damage, stroke or death. The inaccuracy in placement may result in spilling into other smaller arteries eg. the meningeal artery or its smaller branches leading to neurological damage to III, IV, V,VI and VII nerves. It may also enter the posterior branches of the ascending pharyngeal artery causing damage to IX, X, XI and XII cranial nerves.

In view of the possible complications that could arise from embolisation, surgical excision and curettage was preferred. Radical resection has also been 
recommended $(3,4)$ but curettage as in this case and other reported cases were also successful $(5,6)$ The latter preserved the form and function of the mandible.

Haemangiomas, because of their tendency to involute, are generally managed conservatively unless they bleed, ulcerate or interfere with function(9). Other less invasive modes of treatment include the use of the sclerosing agent ( sodium tetradecyl sulphate). It works equally well with minimal local and systemic effects and therefore well recommended(10). Platinum mini spiral can also be used to embolize to reduce the size of the tumour prior to surgery(11).
In conclusion, it is recommended that for medium sized haemangiomas such as in this case, in the absence of facilities to embolize it, surgical excision may be safely carried out provided proper precautions have been taken into account. An angiogram is of utmost important in determining the feeding vessels and therefore crucial before any surgical procedure is attempted.

\section{REFERENCES} 1. Van den Akker HP, Kuiper L, Peters F L. Embolisation of an arterio-venous malformation of the mandible. J Oral Maxillofac Surg
$1987 ; 45: 255-60$.

2. William MD, Pearson MH, Thomas FD. Arterial embolisation of a facial haemangioma. Br Dent J 1992: 173; 102-4.

3. Babin RW, Osbon DB, Khangure MS. Arteriovenous malformations of the mandible. Otolaryngol Head Neck Surg 1983; 91; 36 71.

4. Anderson JH, Grisius RJ, McKean TW. Arteriovenous malformation of the mandible. Oral Surg 1981; 52: 118-25.

5. Kelly DE, Terry BC, Small EW. Arteriovenous malformation of the mandible: report of case. J Oral Surg 1977; 35 : $387-93$.

6. Gallagher DM, Hilley D, Epker BN. Surgical treatment of an arteriovenous malformation of the mandible in a child. A case report. J Maxillofac Surg 1983; 11: 279-83.

7. Hashimoto $Y$, Matsushiro $K$, Nagaki $M$ and Tanioka $H$. Therapeutic embolization for vascular lesion of the head and neck, Report of two cases. Int J. Oral Maxillofac. Surg 1989: 18; 47-9.

8. Berenstein A, Kriecheff I.Catheterandmaterialselectionfortransarterial embolization; Technical Consideration (II). Material Radiology 1979: 132; 631-9.

9. Wisnicki Л. Haemangiomas and vascular malformations- An overview. Ann Plast Surg 1984; 12: $41-59$.

10. Baurmash $\mathrm{H}$, DeChiara S. A conservative approach to the management of orofacial vascular lesion in infant and children: report of cases. J Oral Maxillofac Surg 1991; 49: 1222-5.

11. Philippou S and Gellrich NC. Haemangiopericytoma of the head and neck region: a clinical and morphological study of three cases. Int J Oral Maxillofac Surg 1992; 21: 99-103. 